Pacific Journal of Mathematics

DERIVATIONS AND COMMUTATIVITY OF RINGS. II

O. Chung, Jung Lur AND ANTHONY N. Richoux 


\title{
DERIVATIONS AND COMMUTATIVITY OF RINGS II
}

\author{
Lung O. Chung, JiANg LuH AND \\ ANTHONY N. RICHOUX
}

Let $R$ be a ring with center $C$, and $\mathscr{F}$ be the additive group of all inner derivations of $R$. An additive group $\mathscr{D}$ of derivations of $R$ is said to be a primary class of derivations of $R$ if (i) for any $\partial \in \mathscr{D}$ and $\delta \in \mathscr{J}$, $[\partial, \delta] \in \mathscr{D}$, (ii) for any $x \in R, \partial x=0$ for all $\partial \in \mathscr{D}$ if and only if $x \in C$, and (iii) for any prime ideal $P$ in $R$ and any $x \in R, \partial x \in P$ for all $\partial \in \mathscr{D}$ if and only if $\delta x \in P$ for all $\delta \in \mathscr{F}$.

Suppose $R$ has a primary class $\mathscr{D}$ of derivations. First we assume, for each $x \in R$ and $\partial \in \mathscr{D}$, there is a $p \in R$ such that $\partial x-(\partial x)^{2} p \in C$. Then all nilpotent elements in $R$ form an ideal $N$ of $R$ and $R / N$ is a subdirect sum of division rings and commutative rings. If $R$ is prime, then $R$ has no nonzero divisors of zero. Next, we assume that, for each $x \in R$ and $\partial \in \mathscr{D}$, there is a polynomial $p(t)$ of $t$ with integral coefficients such that $\partial x-(\partial x)^{2} p(\partial x) \in C$ or, for each $x \in R$ and $\partial \in \mathscr{D}$, there is a $p \in C$ such that $\partial x-(\partial x)^{2} p \in C$. Then $\partial x \in C$ for all $x \in R$ and $\partial \in \mathscr{D}$. If $R$ is prime, then $R$ is necessarily commutative.

1. Introduction. In a previous paper [1], the authors extended the concept of inner derivation to the concept of primary class of derivations for rings and generalized several commutativity theorems given by Wedderburn, Jacobson, Kaplansky, Herstein, Ligh, Putcha, Wilson and Yaqub. Let $R$ be a ring having a primary class $\mathscr{D}$ of derivations whose definitions and basic properties will be recalled later. Among others, the following results were proved:

(1) Suppose, for each $x \in R$ and $\partial \in \mathscr{D}$, there is a $p \in R$ which depends upon $x$ and $\partial$, such that $\partial x=(\partial x)^{2} p$. Then the nilpotent elements in $R$ form an ideal $N$ in $R$, and $R / N$ is a subdirect sum of division rings and commutative rings.

(2) Suppose, for each $x \in R, \partial \in \mathscr{D}$ such a $p$ is a polynomial of $\partial x$ with integral coefficients. Then $R$ is commutative.

(3) Suppose, for each $x \in R, \partial \in \mathscr{D}$ such a $p$ described in (1) is central. Then $R$ is a commutative.

The purpose of this paper is to generalize these results further by relaxing the condition " $\partial x=(\partial x)^{2} p$. " We will consider the condition " $\partial x-(\partial x)^{2} p \in C$, the center of $R^{\prime \prime}$ instead. More precisely we will consider rings $R$ having a primary class of derivations which satisfies one of the following conditions: 
(AC) For each $x \in R$ and $\partial \in \mathscr{D}$, there is a $p=p(x, \partial) \in R$ such that $\partial x-(\partial x)^{2} p \in C$;

(BC) For each $x \in R$ and $\partial \in \mathscr{D}$, there is a polynomial $p(t)$ of $t$ with integral coefficients such that $\partial x-(\partial x)^{2} p(\partial x) \in C$;

(CC) For each $x \in R$ and $\partial \in \mathscr{D}$, there is a $p \in C$ each that $\partial x-$ $(\partial x)^{2} p \in C$.

We will show that a prime ring satisfying condition (AC) has no nonzero divisors of zero and consequently, for any ring $R$ satisfying the condition (AC), the prime radical $N$ of $R$ consists of all nilpotent elements of $R$ and $R / N$ is a subdirect sum of division rings and commutative rings. A prime ring satisfying the condition (BC) or (CC) must be commutative. In any ring $R$ satisfying the condition (BC) or (CC), $\partial x$ is central for all $\partial \in \mathscr{D}$ and $x \in R$. These not only generalize some results in [1] but also generalize a result of Martindale [9].

Finally, we will exhibit an example of a primary class of derivations in which not all derivations are inner. This gives an affirmative answer to a problem raised in [1]. Complementing other examples in [1], this shows that the concept of a primary class of derivations is a much more general concept than that of inner derivations.

2. Preliminaries. Let $R$ be a ring, $C$ be its center and $\mathscr{J}$ be the additive group of all inner derivations of $R$. An additive group $\mathscr{D}$ of derivations of $R$ is said to be a primary class of derivations of $R$ if

(i) For any $\partial \in \mathscr{D}$ and $\delta \in \mathscr{F},[\partial, \delta] \in \mathscr{D}$;

(ii) For any $x \in R, \partial x=0$ for all $\partial \in \mathscr{D}$ if and only if $x \in C$;

(iii) For any prime ideal $P$ in $R$ and any $x \in R, \partial x \in P$ for all $\partial \in \mathscr{D}$ if and only if $\delta x \in P$ for all $\delta \in \mathscr{F}$.

It is clear that $\mathscr{F}$ itself is a primary class of derivations of $R$, (iii) $\Rightarrow$ (ii) for any prime ring $R$, and (i) is true if and only if, for any $\partial \in \mathscr{D}$ and $x \in R$, the inner derivation $\delta_{\partial x}: r \rightarrow[\partial x, r]$ lies in $\mathscr{D}$. It is also easy to see that, for all $\partial \in \mathscr{D}, \partial x \in N$, the radical of $R$, if and only if, for all $\delta \in \mathscr{F}, \delta x \in N$. For convenience, we denote by $\mathscr{D}(R)$ the set of elements $\partial x$ where $\partial \in \mathscr{D}$ and $x \in R$.

Proposition 2.1. Let $R$ be a ring $R^{*}$ be a prime ring which is a homomorphic image of $R$ under a homomorphism $\phi$. Suppose $\mathscr{D}$ is a primary class of derivations of $R$. For each $\partial \in \mathscr{D}$, define $\partial^{*}: R^{*} \rightarrow R^{*}$ by $\partial^{*}(\phi(x))=\phi(\partial x)$ for all $x \in R$. Then the set $\mathscr{D}^{*}$ of all $\partial^{*}$, where $\partial \in \mathscr{D}$, forms a primary class of derivations of $R^{*}$.

Proof. See [1]. 
CoROLlaRY 2.2. Let $R$ be a ring and $N$ be the prime radical of $R$. Suppose $\mathscr{D}$ is a primary class of derivations of $R$. For each $\partial \in \mathscr{D}$, define $\partial^{*}: R / N \rightarrow R / N$ by $\partial^{*}(x+N)=\partial z+N$ for all $x \in R$. Then the set $\mathscr{D}^{*}$ of all $\partial^{*}$, where $\partial \in \mathscr{D}$, forms a primary class of derivations of $R / N$.

In [1], we considered a ring $R$ having a primary class $\mathscr{D}$ of derivations and satisfying one of the following conditions:

(A) For each $x \in R$ and $\partial \in \mathscr{D}$, there is a $p=p(x, \partial) \in R$ such that $\partial x=(\partial x)^{2} p$

(B) For each $x \in R$ and $\partial \in \mathscr{D}$, there is a polynomial $p(t)$ of $t$ with integral coefficients such that $\partial x=(\partial x)^{2} p(\partial x)$;

(C) For each $x \in R$ and $\partial \in \mathscr{D}$, there is a $z \in C$ such that $\partial x=$ $(\partial x)^{2} z$.

The following theorems have been proved in [1]:

THEOREM 2.3. Suppose $R$ is a prime ring having a-primary class $\mathscr{D}$ of derivations which satisfy the condition (A). Then $R$ is either a commutative ring or a division ring.

THEOREM 2.4. Suppose $R$ is a ring having a primary class $\mathscr{D}$ of derivations which satisfies the condition (A). Then $R / N$ is a subdirect sum of division rings and commutative rings.

THEOREM 2.5. Suppose $R$ is a ring having a primary class $\mathscr{D}$ of derivations which satisfies the condition (B). Then $R$ is commutative.

THEOREM 2.6. Suppose $R$ is a ring having a primary class $\mathscr{D}$ of derivations which satisfies the condition (C). Then $R$ is commutative.

As we pointed out earlier, since commutators are images of inner derivations these theorems generalize some results given by Herstein [3], Ligh [8], Putcha, Wilson and Yaqub [11].

Throughout this paper, $R$ denotes a ring with center $C$, $\mathscr{z}$ the ring of integers, $\mathscr{Z}^{+}$the set of all positive integers and $\mathscr{Q}$ the field of rational numbers. For $x \in R, \delta_{x}$ denotes the inner derivation $y \mapsto$ $[x, y]$ for all $y \in R$.

3. Primary classes satisfying (AC).

Definition 3.1. Let $R$ be a ring. An element $x \in R$ has the 
property $\mathscr{P}$ if, for any nonzero ideal $I$ of $R$, there exists $n=$ $n(I) \in \mathscr{Z}^{+}$such that $x^{n} \in I$.

The following lemma which can be found in $[4, \mathrm{p} .53]$ will be used later.

LEMma 3.1. A ring $R$ having no nonzero nil ideals is a subdirect sum of prime rings $R_{\alpha}$, where, for each $\alpha, R_{\alpha}$ has a nonnilpotent element having property $\mathscr{P}$.

LEMma 3.2. Let $R$ be a prime ring and $\partial$ be a derivation on $R$. Suppose $\partial x \in C$, the center of $R$, for all $x \in R$. Then either $\partial=0$ or $R$ is commutative.

Proof. Let $y \in R$. For any $x \in R, 0=[\partial(x y), y]=[\partial x y+x \partial y, y]=$ $\partial y[x, y]$. Since $\partial y \in C$ and $R$ is prime, either $\partial y=0$ or $y \in C$. Let $T=\{y \in R \mid \partial y=0\}$. Then $R=C \cup T$, a union of two additive subgroups of the additive group $R$. So either $C=R$ or $T=R$. That is, either $R$ is commutative or $\partial=0$.

A well known fact in ring theory says that, in a 2-torsion free semiprime ring $R$, if $a$ commutes with $[a, x]$ for all $x \in R$ then $a$ lies in the center of $R$. In view of this, we would like to point out that in the hypothesis of Lemma 3.2, the condition that $R$ being prime is essential. This can be seen from the following example.

EXAMPLE. Let $R=A \oplus F[x]$, where $A$ is a noncommutative prime ring and $F[x]$ is the polynomial ring over a field $F$. Let $\partial: R \rightarrow R$ be defined by $\partial(a, f(x))=\left(0, f^{\prime}(x)\right)$ for $a \in A, f(x) \in F[x]$, where $f^{\prime}(x)$ denotes the derivative of $f(x)$. Then $R$ is semiprime, $\partial$ is a derivation on $R$, and $\partial R$ is contained in the center of $R$. However, $\partial \neq 0$ and $R$ is not commutative.

Throughout the balance of this section, we assume $R$ is a ring having a primary class $\mathscr{D}$ of derivations which satisfies the condition (AC). Let us start with

Lemma 3.3. Suppose $R$ is prime. If $\partial x \in \mathscr{D}(R)$ is nilpotent, then $\partial x=0$.

Proof. Let $p \in R$ be such that $\partial x-(\partial x)^{2} p \in C$. Assume $(\partial x)^{n}=0$ but $(\partial x)^{n-1} \neq 0$ for $n>1$. Since $\left[\partial x-(\partial x)^{2} p, \partial x\right]=0$, we obtain $(\partial x)^{3} p=(\partial x)^{2} p \partial x$, and consequently $\left(\partial x-(\partial x)^{2} p\right)^{n-1}=(\partial x)^{n-1} \in C$. Since zero is the only central nilpotent element in a prime ring, we have $(\partial x)^{n-1}=0$, a contradiction. 
Lemma 3.4. Suppose $R$ is prime and $a, z, w \in R, \partial, \partial_{1} \in \mathscr{D}$. If $a^{2}=0$ and $\left[\partial z+\partial_{1} w, a\right]=0$, then $a\left[\partial z+\partial_{1} w, y\right]=0$ for all $y \in R$.

Proof. Note that, since $\delta_{\partial z}+\delta_{\partial_{1} w} \in \mathscr{D}$, for any $y \in R,\left[\partial z+\partial_{1} w, y\right]=$ $\left(\partial_{\partial z}+\partial_{\partial_{1} w}\right) y \in \mathscr{D}(R)$. Since $a\left[\partial z+\partial_{1} w, y\right] a=\left[\partial z+\partial_{1} w, a y a\right] \in \mathscr{D}(R)$ is nilpotent, $\left[\partial z+\partial_{1} w, a y a\right]=0$ by Lemma 3.3. It follows that $\left[\partial z+\partial_{1} w, a y\right]=a\left[\partial z+\partial_{1} w, y\right] \in \mathscr{D}(R)$ is nilpotent and hence, by Lemma 3.3 again, $a\left[\partial z+\partial_{1} w, y\right]=0$ as we desired.

LemMA 3.5. Suppose $R$ is prime and $a \in R$. If $a^{2}=0$ then $\partial a \in C$ for all $\partial \in \mathscr{D}$.

Proof. From $0=\partial a^{2}=\partial a a+a \partial a$, we have $[\partial a, a]^{2}=0$. Since $[\partial a, a] \in \mathscr{D}(R)[\partial a, a]=0$ by Lemma 3.3.

Since $[\partial a, a]=0$, for any $y \in R,[\partial a, a y]=0$ by Lemma 3.4. Thus, $0=\partial[\partial a, a y]=\partial a[\partial a, y]+a\left[\partial^{2} a, y\right]$. On the other hand $0=$ $\partial[\partial a, a]=\left[\partial^{2} a, a\right]$ implies that $a\left[\partial^{2} a, y\right]=0$ by Lemma 3.4. Hence $0=\partial a[\partial a, y]$ and likewise $0=[\partial a, y] \partial a$. By expansion of $[\partial a, y]^{4}$, we obtain $[\partial a, y]^{4}=0$. Therefore, by Lemma 3.3, $[\partial a, y]=0$.

LEMmA 3.6. Suppose $R$ is prime and $a \in R$. If $a^{2}=0$ and $[\partial z, a]=0$, where $\partial \in \mathscr{D}, z \in R$, then $\partial_{1} a \partial z \in C$ for all $\partial_{1} \in \mathscr{D}$.

Proof. By Lemma 3.4, $a[\partial z, y]=0$. Thus $0=\partial_{1}(a[\partial z, y])=$ $\partial_{1} a[\partial z, y]+a\left[\partial_{1} \partial z, y\right]+a\left[\partial z, \partial_{1} y\right]$. On the other hand, since $[\partial z, a]=0$, $0=\partial_{1}[\partial z, a]=\left[\partial_{1} \partial z, a\right]+\left[\partial z \partial_{1}, a\right]=\left[\partial_{1} \partial z, a\right]$ by Lemma 3.5. By Lemma 3.4, $a\left[\partial_{1} \partial z, y\right]=0$, and $a\left[\partial z, \partial_{1} y\right]=0$. Thus, $\partial_{1} a[\partial z, y]=0$ for all $y \in R$, i.e., $\left[\partial_{1} a \partial z, y\right]=0$ for all $y \in R$. Hence $\partial_{1} a \partial z \in C$.

LEMMA 3.7. Suppose $R$ is prime and $a \in R$. If $a^{2}=0$, then $\partial_{2} a \partial_{1} \partial y \in C$ for all $\partial, \partial_{1}, \partial_{2} \in \mathscr{D}$ and $y \in R$.

Proof. By Lemma 3.5, $[\partial y, a] \in C$ which yields $\left[\partial_{1} \partial y, a\right]=$ $\partial_{1}[\partial y, a]=0$. It follows immediately from Lemma 3.6 that $\partial_{2} a \partial_{1} \partial y \in C$.

Lemma 3.8. Suppose $R$ is prime and $a \in R$. If $a^{2}=0$, then $\partial_{1} a \partial^{2} y=0$ for all $\partial, \partial_{1} \in \mathscr{D}$ and $y \in R$.

Proof. Since by Lemma 3.5, $0=\partial_{1} \partial\left(a^{2}\right)=2 \partial_{1} a \partial a$, we get $\partial\left(\partial_{1} a \partial(a y)\right)=\partial_{1} a \partial^{2}(a y)=\partial_{1} a\left(2 \partial a y+a \partial^{2} y\right)=a \partial_{1} a \partial^{2} y$ whose square is zero by Lemma 3.7. Hence $a \partial_{1} a \partial^{2} y=0$. It follows that $0=$ $\partial_{1}\left(a \partial_{1} a \partial^{2} y\right)=\left(\partial_{1} a\right)^{2} \partial^{2} y+a \partial_{1} a \partial_{1} \partial^{2} y=\left(\partial_{1} a\right)^{2} \partial^{2} y$ by Lemma 3.7. Hence 
$\left(\partial_{1} a \partial^{2} y\right)^{2}=0$. By noting that $\partial_{1} a \partial^{2} y=\partial\left(\partial_{1} a \partial y\right) \in \mathscr{D}(R)$, we obtain $\partial_{1} a \partial^{2} y=0$.

Lemma 3.9. Suppose $R$ is prime, $\partial, \partial_{1} \in \mathscr{D}$, and $a, y \in R$. If $a^{2}=0$ and $(\partial a)^{2} \partial_{1} y \in C$ then $\partial a \partial_{1} y \in C$.

Proof. Since $(\partial a)^{2}\left[\partial_{1} y, z\right]=\left[(\partial a)^{2} \partial_{1} y, z\right]=0,\left(\partial a\left[\partial_{1} y, z\right]\right)^{2}=0$. Note that $\partial a\left[\partial_{1} y, z\right] \in \mathscr{D}(R)$. Hence $\partial \alpha\left[\partial_{1} y, z\right]=0$ for all $z \in R$, i.e., $\partial a \partial_{1} y \in C$.

Lemma 3.10. Suppose $R$ is prime and $a \in R$. If $a^{2}=0$ then $\partial_{2} a\left(\partial_{1} a \partial y+\partial a \partial_{1} y\right) \in C$ for all $\partial, \partial_{1}, \partial_{2} \in \mathscr{D}$ and $y \in R$.

Proof. Let $u=\partial_{2} a\left(\partial_{1} a \partial y+\partial a \partial_{1} y\right)$. Then $u+a \partial_{2} a \partial_{1} \partial y=\partial_{2} a \partial_{1} \partial(a y)$ which is central by Lemma 3.7. Since $\partial_{2} a \partial_{1} \partial y \in C,[u, a]=0$. Note that $u=\partial\left(\partial_{2} a \partial_{1} a y\right)+\partial_{1}\left(\partial_{2} a \partial a y\right)$. Thus by Lemma 3.4, for all $z \in R$, $a[u, z]=0$, from which $0=\partial_{2}(a[u, z])=\partial_{2} a[u, z]+a\left[\partial_{2} u, z\right]$. The last term is zero since $\partial_{2} u=\partial_{2} a\left(\partial_{1} a \partial_{2} \partial y+\partial a \partial_{2} \partial_{1} y\right) \in C$ by Lemma 3.7. Thus, $\partial_{2} a[u, z]=0$. Suppose $u \neq 0$. Then $\partial_{2} a \neq 0$ and $[u, z]=0$ for all $x \in R$. Hence $u \in C$ as we desired.

Lemma 3.11. Suppose $R$ is prime and $a \in R$. If $a^{2}=0$ then $\partial_{1} a \partial_{1} \partial y=0$ for all $\partial, \partial_{1} \in \mathscr{D}$ and $y \in R$.

Proof. By Lemma 3.10, $\left(\partial_{1} \alpha\right)^{2} \partial y+\partial_{1} \alpha \partial \alpha \partial_{1} y \in C$. Applying $\partial_{1}$ and using Lemma 3.8, we get $\left(\partial_{1} a\right)^{2} \partial_{1} \partial y=0$. Thus, $\left(\partial_{1} a \partial_{1} \partial y\right)^{2}=0$. Since $\partial_{1} a \partial_{1} \partial y=\partial_{1}\left(\partial_{1} a \partial y\right) \in \mathscr{D}(R), \partial_{1} a \partial_{1} \partial y=0$.

Lemma 3.12. Suppose $R$ is prime and $a \in R$. If $a^{2}=0$ then $\partial a \partial y \in C$ for all $\partial \in \mathscr{D}$ and $y \in R$.

Proof. By Lemma 3.5, $[\partial y, a] \in C$. So $0=\partial_{1}[\partial y, a]=\left[\partial_{1} \partial y, a\right]$. By Lemma 3.11, $0=\partial a \partial \partial_{1}(a y)=\partial a\left(\partial a \partial_{1} y+\partial_{1} a \partial y+a \partial \partial_{1} y\right)=\partial a\left(\partial a \partial_{1} y+\right.$ $\left.\partial_{1} \alpha \partial y\right)$. Consequently $\partial_{1}(\partial a \partial(y a))=\partial a\left(\partial_{1} \partial y a+\partial_{1} y \partial a+\partial y \partial_{1} a\right)=\partial a \partial_{1} \partial y a$. Since $\partial a \partial_{1} \partial y a \in \mathscr{D}(R)$ and its square is zero by Lemma 3.6, $\partial a \partial_{1} \partial y a=0$ by Lemma 3.3. It follows that $\partial a \partial_{1} \partial y=0$ because $\partial a \partial_{1} \partial y \in C$ and $R$ is prime. That is, $\partial_{1}(\partial a \partial y)=0$ for all $\partial_{1} \in \mathscr{D}$. Hence $\partial a \partial y \in C$.

Now we are in a position to prove one of our main results.

Proposition 3.13. Suppose $R$ is a prime ring having a primary class of derivations $\mathscr{D}$ which satisfies the condition (AC). Then $R$ has no nonzero nilpotent elements and hence $R$ has no zero divisors.

Proof. Let $0 \neq b \in R$ be a nilpotent element and $b^{n}=0, b^{n-1} \neq 0$ 
where $n>1$. We set $a=b^{n-1}$. Then $a^{2}=0$. By Lemma 3.12 $\partial a \partial(a y) \in C$ for all $\partial \in \mathscr{D}$ and $y \in R$. So $\left[(\partial a)^{2} y+\partial a a \partial y, a\right]=0$. Since $\partial a a \partial y=a \partial a \partial y$ commutes with $a$, we have $a(\partial a)^{2} y a=-\left[(\partial a)^{2} y, a\right] a=0$. Note that $y$ is arbitrary and $R$ is prime. Thus $a(\partial a)^{2}=0$. Again by the primeness of $R$ and the fact that $\partial a \in C$, we obtain $(\partial a)^{2}=0$ and hence by Lemma 3.3, $\partial a=0$ for all $\partial \in \mathscr{D}$. Therefore $a \in C$ which yields $a=0$, i.e., $b^{n-1}=0$, a contradiction. This completes the proof.

THEOREM 3.14. Let $R$ be a ring having a primary class $\mathscr{D}$ of derivations which satisfies the condition (AC). Then the nilpotent elements in $R$ form an ideal in $R$.

Proof. $R / N$ is a subdirect sum of prime rings $R_{\alpha}, \alpha \in I$, where $N$ denotes the prime radical of $R$. According to Proposition 2.1, each $R_{\alpha}$ has a primary class $\mathscr{D}$ of derivations which also satisfies the condition (AC). By Proposition 3.13, each $R_{\alpha}$ has no nonzero nilpotent elements. Let $\psi_{\alpha}$ be the epimorphism of $R$ into $R_{\alpha}$ such that $N=\bigcap_{\alpha \in I} \operatorname{Ker} \psi_{\alpha}$. Then for any nilpotent element $x \in R, \psi_{\alpha}(x)=0$, i.e., $x \in \operatorname{Ker} \psi_{\alpha}$ for all $\alpha \in I$. So $x \in N$. Therefore $N$ contains the set of all nilpotent elements in $R$.

Proposition 3.15. Let $R$ be a subdirectly irreducible prime ring having a primary class $\mathscr{D}$ of derivations which satisfies the condition (AC). Then $R$ is a division ring.

Proof. Let $S$ be the minimal ideal of $R$ and $C$ be the center of $R$.

Suppose $S \subseteq C$. Let $0 \neq x \in S$. Then $x S=S$ and $x e=x$ for some $e \in S$. It can be seen easily that $e$ is an identity of $R$ and $S=$ $R=C$ and hence $R$ is a field.

Thus we may assume now that $S \nsubseteq C$. There exist $\partial \in \mathscr{D}$ and $x \in S$ such that $\partial x \neq 0$. By condition (AC) there exists $p \in R$ such that $u=\partial x-(\partial x)^{2} p \in C$. We claim that $R$ has identity 1 and that there exists $v_{0} \in S$ such that $\partial v_{0}$ is invertible. Indeed, if $u=0$ then, by Proposition 3.13, $\partial x p$ is an identity element in $R$ and $\partial x$ is invertible. Suppose $u \neq 0$. Then $u S=S$ and $u v=x$ for some $v \in S . \quad \partial x=\partial(u v)=u \partial v=\partial x(\partial v-\partial x p \partial v)$ implies that $\partial v-\partial x p \partial v$ is an identity element in $R$ and $\partial v$ is invertible by Proposition 3.13.

Now we assert that $R=S$. For otherwise there would exist a prime ideal $P$ of $R$ such that $S \subseteq P$ and $P \neq R$. Since $v_{0} \in S$ and $\delta v_{0} \in P$ for all inner derivations $\delta$ of $R, \partial v_{0} \in P$. This would imply that $P$ contains the identity 1 of $R$, a contradiction. Thus $R=S$. 
In view of Theorem 2.4 , to show that $R$ is a division ring it suffices to show the condition (A) holds for $R$. In fact, let $\partial \in \mathscr{D}$ and $y \in R$ and $u=\partial y-(\partial y)^{2} q \in C$ where $q \in R$. Suppose $u \neq 0$. Then since $R=S, u R=R$ and $u$ is invertible. It follows that $\partial y$ is invertible. Hence $\partial y$ satisfies the conditions (A). This completes the proof.

In preparation for the proof that $R / N$ is a subdirect sum of commutative rings and division rings, we first establish the following lemmas.

LEMMA 3.16. Suppose $R$ is a prime and suppose there is a nonzero element in $R$ which has the property $\mathscr{P}$ (see Definition 3.1). Then there exists a nonzero central element in $R$ which also has the property $\mathscr{P}$.

Proof. Let $0 \neq a \in R$ have the property $\mathscr{P}$. Suppose $a \notin C$, the center of $R$. Then there is a $\partial \in \mathscr{D}$ such that $\partial a \neq 0$. By the condition (AC), for some $p \in R, u=\partial a-(\partial a)^{2} p \in C$.

Case 1. $u=0$. Using Proposition 3.13, one can verify that $\partial a p$ is an identity 1 of $R$. Suppose $R$ is not simple. There exists a maximal ideal $M$ of $R$. $R / M$ is a subdirectly irreducible prime ring which, by Proposition 2.1, has a primary class of derivations satisfying the condition (AC). By Proposition $3.15, R / M$ is a division ring. Since $a^{n} \in M$ for some positive integer $n, a \in M$. Since $\delta a \in M$ for all inner derivations $\delta$ of $R, \partial a \in M$. Hence $1 \in M$, a contradiction. Therefore $R$ must be simple and $1 \in C$ has the property $\mathscr{P}$.

Case 2. $u \neq 0$. Let $I$ be an arbitrary nonzero ideal in $R$. We claim that for some $n \in \mathscr{Z}^{+},(\partial a)^{n} \in I$. Suppose not. Then, by Zorn's lemma, there exists a prime ideal $M$ in $R$ containing $I$ such that $(\partial a)^{m} \notin M$ for all $m \in \mathscr{Z}^{+}$. Since $R / M$ is a prime ring having a primary class of derivations which satisfies the condition (AC), by Proposition $3.13 R / M$ has no nonzero nilpotent elements. But $a^{m} \in M$ for some $m \in \mathscr{Z}^{+}$. So $a \in M$ and consequently $\partial a \in M$, a contradiction. Hence $(\partial a)^{n} \in I$ for some $n \in \mathscr{Z}^{+}$. By noting that $\left[\partial a-(\partial a)^{2} p, \partial a\right]=0$, we obtain $(\partial a)^{2} p \partial a=(\partial a)^{3} p$ which implies that $u^{n}=\left(\partial a-(\partial a)^{2} p\right)^{n} \in I$. Thus $u$ is a central element in $R$ which has the property $\mathscr{P}$.

LEMMA 3.17. Suppose $R$ is prime and suppose there exists $a \neq 0$ in $R$ which has the property $\mathscr{P}$. Then $R$ is either a commutative ring or a division ring. 
Proof. Suppose $R$ is not commutative. By Lemma 3.2, there exist $\partial \in \mathscr{D}$ and $x \in R$ such that $\partial x \notin C$. By Lemma 3.16, there exists $a \in C, a \neq 0$, which has the property $\mathscr{P}$. Let $I$ be a nonzero ideal in $R$ and $a^{n} \in I$, where $n \in \mathscr{Z}^{+}$. Noting that $a^{n} \partial x=\partial\left(a^{n} x\right) \in$ $\mathscr{D}(R)$ we have, by the condition (AC), $a^{n} \partial x-\left(a^{n} \partial x\right)^{2} p \in C$ for some $p \in R$. It follows that $a^{n}\left(\partial x-a^{n}(\partial x)^{2} p\right) \in C$ and hence $\partial x-a^{n}(\partial x)^{2} p \in C$. Since $\partial x \notin C,[\partial x, y] \neq 0$ for some $y \in R$. But from $\left[\partial x-a^{n}(\partial x)^{2} p, y\right]=0$, we obtain $0 \neq \partial x y-y \partial x=a^{n}(\partial x)^{2} p y-y a^{n}(\partial x)^{2} p \in I$. This is true for all nonzero ideals $I$ in $R$. Hence $R$ is subdirectly irreducible. By Proposition $3.15, R$ is a division ring.

The following theorem is a generalization of Theorem 2.4.

THEOREM 3.18. Let $R$ be a ring having a primary class $\mathscr{D}$ of derivations which satisfies the property (AC). Let $N$ be the prime radical of $R$. Then $R / N$ is a subdirect sum of commutative rings and division rings.

Proof. $R / N$ is a subdirect sum of prime rings $R_{\alpha}, \alpha \in A$. Each $R_{\alpha}$ has a primary class of derivations which satisfies the condition (AC) by Proposition 2.1. By Theorem 3.14, $R / N$ has no nonzero nilpotent elements and hence, by Lemma 3.1, we may assume that each $R_{\alpha}$ has a nonzero element which has the property $\mathscr{P}$. Thus, by Lemma 3.17 , each $R_{\alpha}$ is either a commutative ring or a division ring.

We should note that if we replace the condition (AC) by the following condition for a ring $R$ :

$(\mathrm{AC})^{\prime}$ For each $\partial \in \mathscr{D}$ and $x \in R$, there is a $p \in R$, such that $\partial x-p(\partial x)^{2} \in C$,

all the above results remain true.

It would be interesting to consider the following condition which is clearly weaker than the condition (AC) for a ring $R$ having a primary class of derivations.

$(\mathrm{AC})^{*}$ There exists a nonnilpotent central element $c \in R$, such that, for each $\partial \in \mathscr{D}$ and $x \in R, c \partial x-(\partial x)^{2} p \in C$ for some $p \in R$. The condition $(\mathrm{AC})^{*}$ is equivalent to the condition (AC) if $R$ is prime. In fact, assuming $R$ is prime and satisfies the condition $(\mathrm{AC})^{*}$, for any $y \in R, c(\partial(c y))-(\partial(c y))^{2} p \in C$ for some $p \in R$ implies $c^{2}\left(\partial y-(\partial y)^{2} p\right) \in C$ which yields $\partial y-(\partial y)^{2} p \in C$.

One would wonder whether Theorem 3.18 will be true if one replaces the condition (AC) by the condition $(\mathrm{AC})^{*}$. The following counter example is suggested by the referee.

Example. Let $F$ be any field, $S$ any ring and $R=F \oplus S$. Let 
$\mathscr{D}$ be the primary class of all inner derivations and $c=(1,0)$. Then the condition $(\mathrm{AC})^{*}$ holds for all $\partial x \in \mathscr{D}(R)$ with $p=(0,0)$.

4. Primary classes satisfying (BC). Throughout this section we assume $R$ is a ring having a primary class $\mathscr{D}$ of derivations which satisfies the condition (BC) defined in $\S 1$.

LEMma 4.1. Suppose $R$ is a division ring and $F$ is the prime field of $R$. If $\partial a \in \mathscr{D}(R)$ is transcendental over $F$ then $\partial a \in C$, the center of $R$.

Proof. Suppose to the contrary that $\partial a \notin C$. Let $u=\partial a-$ $(\partial a)^{2} p(\partial a) \in C$, where $p(t) \in \mathscr{z}[t]$. Since $u$ is transcendental over $F$ and $F[u]$ is an Euclidean domain there are infinitely many prime elements in $F[u]$, say $\alpha_{1}, \alpha_{2}, \alpha_{3}, \cdots$. By the condition (BC) and the fact that $\alpha_{i} \partial a=\partial\left(\alpha_{i} a\right) \in \mathscr{D}(R)$, there exists $p_{i}(t) \in F[u][t]$ of least degree such that $\alpha_{i} \partial a-\left(\alpha_{i} \partial a\right)^{2} p_{i}\left(\alpha_{i} \partial a\right) \in C$. Here we would note that $p_{i}(t) \neq 0$ for otherwise $\alpha_{i} \partial a \in C$ would imply $\partial a \in C$. Now, we claim that $\operatorname{deg} p_{i} \leqq \operatorname{deg} f$ where $f(t)=t-t^{2} p(t)-u \in F[u][t]$. Indeed, let $n=\operatorname{deg} f$ and $m_{i}=\operatorname{deg} p_{i}$. Suppose to the contrary $m_{i}>n$ for some $i$. Let $f_{i}(t)=\alpha_{i}^{n} f\left(\alpha_{i}^{-1} t\right) \in F[u][t]$ and let $\beta$ and $\gamma$ be the leading coefficients of $f_{i}(t)$ and $p_{i}(t)$ respectively. Let $g(t)=p_{i}(t)-$ $\gamma \beta^{-1} t^{m_{i}-n} f_{i}(t)$. Note that since $\beta \in F, g(t) \in F[u][t]$. Also $g(t) \neq 0$ because $f_{i}\left(\alpha_{i} \partial \alpha\right)=0$ and $g\left(\alpha_{i} \partial \alpha\right)=p_{i}\left(\alpha_{i} \partial \alpha\right) \neq 0$. Since deg $g<m_{i}$ and $\alpha_{i} \partial a-\left(\alpha_{i} \partial a\right)^{2} g\left(\alpha_{i} \partial a\right)=\alpha_{i} \partial a-\left(\alpha_{i} \partial a\right)^{2} p_{i}\left(\alpha_{i} \partial a\right) \in C$, we arrive a contradiction of the choice of $p_{i}$. Hence $m_{i} \leqq n$ for all $i$ as we desired. Thus, we obtain a sequence of polynomials $g_{i}(t) \in F[u][t], g_{i}(t)=$ $\sum_{j=1}^{n_{i}} \beta_{i j} t^{j}, \beta_{1 n_{i}} \neq 0$, which satisfies the following properties:

(i ) $\alpha_{i}^{j} \mid \beta_{i j}$ and $\alpha_{i}^{2} \nmid \beta_{i 1}$ in $F[u]$;

(ii) $g_{i}(\partial a) \in C$;

(iii) $n_{i} \leqq n+2$.

In fact, $g_{i}(t)=\alpha_{i} t-\left(\alpha_{i} t\right)^{2} p_{i}\left(\alpha_{i} t\right), i=1,2, \cdots$, is a such sequence. Without loss of generality, we may choose each $g_{i}(t)$ to be polynomial over $F[u]$ with least degree satisfying the conditions (i), (ii) and (iii). Since $n_{i} \leqq n+2$ for all $i$, there is a subsequence of $\left\{g_{i}(t)\right\}$ which again may be called $g_{1}(t), g_{2}(t), \cdots$ having the same least degree $m$ say. Suppose $m>1$. Let $h_{i}(t)=\beta_{1 m} g_{i}(t)-\beta_{i m} g_{1}(t)=$ $\sum_{j=1}^{m-1} \gamma_{i j} t^{j}$, where $\gamma_{i j}=\beta_{1 m} \beta_{i j}-\beta_{i m} \beta_{1 j}$. Then clearly $h_{i}(t) \in F[u][t]$ and $\operatorname{deg} h_{i}<m$ and $\alpha_{i}^{j} \mid \gamma_{i j}$ for $j=1,2, \cdots, m-1$. If $\alpha_{i}^{2} \mid \gamma_{i 1}=$ $\beta_{1 m} \beta_{i 1}-\beta_{i m} \beta_{11}$, then since $\alpha_{i}^{2} \mid \beta_{i m}$ and $\alpha_{i}^{2} \nmid \beta_{i 1}$ we must have $\alpha_{i} \mid \beta_{1 m}$. But $\beta_{1 m}$ has only a finite number of prime divisors in $F[u]$, we must have an infinite subsequence $\left\{\alpha_{i_{k}}\right\}_{k=1}^{\infty}$ of $\left\{\alpha_{i}\right\}_{i=1}^{\infty}$ for which $\alpha_{i_{k}}^{2} \nmid \gamma_{i_{k}}$, $k=1,2, \cdots$. This indivisibility also guarantees that $\operatorname{deg} h_{i_{k}} \geqq 1$ for all $k$. Now, since $1 \leqq \operatorname{deg} h_{i_{k}}<m$ and the sequence $\left\{h_{i_{k}}\right\}$ also satisfies 
the conditions (i), (ii) and (iii), this contradicts the minimality of $m$. Thus, $m=1$ and hence $\partial a \in C$. This completes the proof.

LEMMA 4.2. Suppose $R$ is a division ring and $F$ is the prime field of $R$. If $\partial a \in \mathscr{D}(R)$ is algebraic over $F$ and if the characteristic of $R$ is zero then $\partial a \in C$.

Proof. We may assume $F$ is the field of rational numbers. Since $\partial a$ is algebraic over $F$, there exists $0 \neq f(t) \in \mathscr{L}[t]$ such that $f(\partial a)=0$. Let $\gamma$ be the leading coefficient of $f(t)$ and $n=\operatorname{deg} f(t)$. Then $\gamma \partial a \in \mathscr{D}(R)$ has a monic minimal polynomial over $\mathscr{z}$. For each prime $p \in \mathscr{\&}$, let $g_{p}(t) \in \mathscr{L}[t]$ be a polynomial of least degree such that $p \gamma \partial a-(p \gamma \partial a)^{2} g_{p}(p \gamma \partial a) \in C$. We will show first that $\operatorname{deg} g_{p} \leqq$ $\operatorname{deg} f$. Let $m=\operatorname{deg} g_{p}$ and $h(t)=\gamma^{n-1} p^{n} f\left(p^{-1} \gamma^{-1} t\right)$. Then clearly $h(t) \in \mathscr{Z}[t]$ is monic and $h(p \gamma \partial a)=0$. Suppose to the contrary that $m \geqq n$. Then $p \gamma \partial \alpha-(p \gamma \partial a)^{2}\left(g_{p}(p \gamma \partial a)-\alpha(p \gamma \partial \alpha)^{m-n} h(p \gamma \partial a)\right) \in C$, where $\alpha$ is the leading coefficient of $g_{p}$, and $g_{p}(t)-\alpha t^{m-n} h(t) \in \mathscr{Z}[t]$ is of degree less than $m$. This contradicts the minimality of $m$. Hence $m \leqq n$.

Now let $f_{1}(t)=\operatorname{irr}(\gamma \partial a, F)$, i.e., $f_{1}(t) \in F[t]$ is irreducible over $F$ and $f_{1}(\gamma \partial \alpha)=0$. For each prime $p \in \mathscr{z}^{+}$, let $f_{p}(t)=\operatorname{irr}(p \gamma \partial a, F)$. Note that $\operatorname{deg} f_{p}=\operatorname{deg} f_{1}$. Let $h_{p}(t)=(p t)^{2} g_{p}(p t)-p t+a_{p}$, where $a_{p}=(p \gamma \partial \alpha)-(p \gamma \partial a)^{2} g_{p}(p \gamma \partial \alpha) \in C$. Then $h_{p}(\gamma \partial a)=0$. Thus for each prime $p \in \mathscr{Z}^{+}$we obtain $h_{p}(t) \in C[t]$ such that all coefficients except the constant term are in $\mathscr{z}$, and $1 \leqq \operatorname{deg} h_{p} \leqq \operatorname{deg} f_{1}+2$. Thus we must have an infinite set of primes $\left\{p_{i} \mid i \in \mathscr{O}^{+}\right\}$such that $\operatorname{deg} h_{p_{i}}=k$ for all $i \in \mathscr{Z}^{+}$and some fixed $k \in \mathscr{F}^{+}$. We obtain a sequence of polynomials $\left\{h_{i}(t)\right\}_{i=1}^{\infty}$ over $C$ having the following properties:

(i) $\operatorname{deg} h_{i}=k$;

(ii) $h_{i}(\gamma \partial a)=0$;

(iii) If $h_{i}(t)=\sum_{j=0}^{k} \alpha_{i j} t^{j}$, then $\alpha_{i j} \in \mathscr{Z}$ and $p_{i}^{j} \mid \alpha_{i j}$ for all $j \geqq 1$, and $p_{i}^{2} \nmid \alpha_{i 1}$ in $\mathscr{z}$.

Without loss of generality, we may choose these $p_{i}$ 's such that the $\operatorname{deg} h_{i}=k$ is least.

Suppose $k>1$. We set $q_{i}(t)=\alpha_{1 k} h_{i}(t)-\alpha_{i k} h_{1}(t)$. Then $\operatorname{deg} q_{i}<k$ and if $q_{i}(t)=\sum_{j=1}^{k-1} \beta_{i j} t^{j}$ then $\beta_{i 0} \in C$ and for $j \geqq 1, \beta_{i j} \in \mathscr{L}, p_{i}^{j} \mid \beta_{i j}$. It is also clear that $q_{i}(\gamma \partial a)=0$. Now if $p_{i}^{2} \mid \beta_{i 1}$, then since $p_{i}^{2} \mid \alpha_{i k}$ (we've assumed $k>1$ ) and $p_{i}^{2} \nmid \alpha_{i 1}$, we must have $p_{i} \mid \alpha_{1 k}$. Since there are only a finite number of divisors of $\alpha_{1 k}$, we can choose an infinite subset of $\left\{p_{i}\right\}_{i=1}^{\infty}$ which again may be called $\left\{p_{i}\right\}_{i=1}^{\infty}$ such that $p_{i}^{2} \nmid \beta_{i 1}$. Note that $\operatorname{deg} q_{i} \geqq 1$. But then since $q_{i}(\gamma \partial a)=0$ and $1 \leqq \operatorname{deg} q_{i}<k$, we can pick an infinite subset of these $q_{i}$ 's all of the same degree. This contradicts the minimality of $k$. Hence $k=1$, and $\gamma \partial a$ satisfies 
a linear polynomial $\alpha t+\beta$ where $\alpha \in F$ and $\beta \in C$. Thus $\partial a \in C$ as we desired.

THEOREM 4.3. Let $R$ be a division ring having a primary class $\mathscr{D}$ of derivations which satisfies the condition (BC). Then $R$ is a field.

Proof. Suppose the characteristic of $R$ is zero. The theorem follows immediately from Lemmas 3.2, 4.1, and 4.2. Thus we may assume that the characteristic of $R$ is $p \neq 0$. Suppose to the contrary that $R$ is not commutative. Then according to Lemma 3.2, for each $\partial \in \mathscr{D}$ where $\partial \neq 0$, there is an $x \in R$ such that $\partial x \notin C$. Here $\partial x$ must be algebraic over the prime field $F$ of $R$ by Lemma 4.1. Since $F$ is finite, $(\partial x)^{p^{k}}=\partial x$ for some $k \in \mathscr{L}^{+}$. By Lemma 3.1.1 in [4] there exists a $y \in R$ such that $y \partial x y^{-1}=(\partial x)^{i} \neq \partial x$ for some $i \in \mathscr{Z}$. Now $0 \neq[\partial x, y] \in \mathscr{D}(R)$ and $[\partial x, y] \partial x=\partial x y \partial x-y(\partial x)^{2}=$ $(\partial x)^{i}[\partial x, y]$. In addition, $[\partial x, y]$ is algebraic over $F$ by Lemma 4.1. Let $S=\left\{\sum_{h, j} \alpha_{h j}(\partial x)^{h}[\partial x, y]^{j} \mid \alpha_{h j} \in F\right\}$. Then $S$ is a finite division ring and hence, by the Wedderburn Theorem, $S$ is a field. Particularly, $[\partial x, y] \partial x=(\partial x)[\partial x, y]$, a contradiction. Hence $R$ is commutative.

As we pointed out earlier, a commutator is simply the image of an element under an inner derivation, and the additive group of all inner derivations on a ring $D$ forms a primary class of derivations. Thus we obtain immediately from Theorem 4.3 the following.

CoRollary 4.4 (Martindale). Suppose $D$ is a division ring such that for each commutator $u \in D, u^{n}-u \in C$, the center of $D$ for some $n \in \mathscr{Z}, n>1$. Then $D$ is a field.

THEOREM 4.5. Let $R$ be an arbitrary ring having a primary class $\mathscr{D}$ of derivations which satisfies the condition (BC). Then $\partial a \in C$ for all $\partial \in \mathscr{D}$ and $a \in R$.

Proof. By Theorems 3.17 and $4.3, R / N$ is a commutative ring where $N$ denotes the prime radical of $R$. Thus, for all $x, y \in R$, $[x, y] \in N$. Noting that $N$ is the intersection of all prime ideals in $R$, we see that $\partial x \in N$, i.e., $\partial x$ is nilpotent for all $\partial \in \mathscr{D}$ and $x \in R$.

Suppose to the contrary that there are an $a \in R$ and $\partial \in \mathscr{D}$ such that $\partial a \notin C$. Since $\partial a$ is nilpotent, $(\partial a)^{n}=0$ for some $n \in \mathscr{L}^{+}, n>1$. By the condition (BC), there is $p(t) \in \mathscr{Z}[t]$ such that $\partial x-(\partial x)^{2} p(\partial x) \in C$. Also, there is an integer $j>1$ such that $(\partial x)^{j},(\partial x)^{j+1}, \cdots,(\partial x)^{n-1} \in C$ but $(\partial x)^{j-1} \notin C$. From $\left(\partial x-(\partial x)^{2} p(\partial x)\right)^{j-1} \in C$, we obtain $(\partial x)^{j-1}+g(\partial x) \in C$ where $g(t) \in \mathscr{Z}[t]$ is a polynomial in which each term is of degree 
greater than $j-1$. Since $g(\partial x) \in C,(\partial x)^{j-1} \in C$ which contradicts the choice of $j$.

The following corollary was conjectured by Herstein and was first proved by Martindale [9].

Corollary 4.6. Suppose $A$ is a ring such that, for each commutator $u \in A, u^{n}-u \in C$, the center of $A$, for some $n \in \mathscr{\gamma}, n>1$. Then every commutator lies in $C$.

THEOREM 4.7. Suppose $R$ is a prime ring having a primary class $\mathscr{D}$ of derivations which satisfies the condition (BC). Then $R$ is commutative.

Proof. This is an immediate consequence of Theorem 4.5 and Lemma 3.2 .

5. Primary classes satisfying (CC). Throughout this section we assume $R$ is a ring having a primary class of derivations which satisfies the condition (CC) defined in $\S 1$. We should note that $(\mathrm{CC}) \Rightarrow(\mathrm{AC})$ and, under the condition $(\mathrm{CC}),(\partial x)^{2} \in C$ if and only if $\partial x \in C$, and $\left[y,(\partial x)^{2}\right]=0$ if and only if $[y, \partial x]=0$.

Lemma 5.1. Suppose $R$ is a division ring. Then $R$ is a field.

Proof. Assume the characteristic of $R$ is two. Suppose to the contrary that $R$ is not commutative. By Lemma 3.2, there exist $\partial \in \mathscr{D}$ and $x \in R$ such that $\partial x \notin C$. By the condition (CC), $\partial x+$ $(\partial x)^{2} p \in C$ for some $p \in C$. It follows that $\partial y+(\partial y)^{2} \in C$ where $y=p x$, and $\partial y \notin C$. Let $\partial_{1}$ be an arbitrary element in $\mathscr{D}$. We shall show $\partial_{1} \partial y=0 . \quad 0=\partial_{1}\left(\partial y+(\partial y)^{2}\right)=\partial_{1} \partial y+\partial_{1} \partial y \partial y+\partial y \partial_{1} \partial y$ yields $\partial_{1} \partial y(1+\partial y)=$ $\partial y \partial_{1} \partial y$, i.e., $\left(\partial_{1} \partial y\right)^{-1} \partial y\left(\partial_{1} \partial y\right)=1+\partial y$. It follows that $\left(\partial_{1} \partial y\right)^{-2} \partial y\left(\partial_{1} \partial y\right)^{2}=$ $\left(\partial_{1} \partial y\right)^{-1}(1+\partial y)\left(\partial_{1} \partial y\right)=1+1+\partial y=\partial y$. So $\left[\partial y,\left(\partial_{1} \partial y\right)^{2}\right]=0$ and hence $\left[\partial y, \partial_{1} \partial y\right]=0$. Consequently, $\partial_{1} \partial y=\partial_{1} \partial y \partial y+\partial y \partial_{1} \partial y=0$. Since this holds for all $\partial_{1} \in \mathscr{D}, \partial y \in C$, a contradiction. Hence $R$ is commutative.

Now we assume the characteristic of $R$ is not two. In view of Lemma 3.2, we need only to show $\mathscr{D}(R) \subseteq C$.

Let $\partial \in \mathscr{D}$.

Case 1. $\partial R \cap C=\{0\}$. Suppose to the contrary that $\partial x \notin C$ for some $x \in R$. By the condition (CC), $\partial x-(\partial x)^{2} p \in C$ for some $0 \neq p \in C$. Thus, $\partial y-(\partial y)^{2} \in C$ where $y=p x$ and $\partial y \notin C$. We claim that $\partial R \leqq$ $C \partial y$. Indeed, suppose $\partial R \cong C \partial y$. Then for all $u, v \in R$, we have 
$0=[\partial(u v), \partial y]=\partial u[v, \partial y]+[u, \partial y] \partial v$. Replacing $u$ by $\partial u$, we get $0=$ $\partial^{2} u[v, \partial y]$. Since $\partial y \notin C, \partial^{2} u=0$ for all $u \in R$, i.e., $\partial^{2}=0$. Thus for $x \in R, \quad 0=\partial^{2}\left(x^{2}\right)=\partial^{2} x x+2(\partial x)^{2}+x \partial^{2} x=2(\partial x)^{2}$ and hence $\partial x=0$, a contradiction. Hence $\partial R \nsubseteq C \partial y$. Now let $\partial z \notin C \partial y$. By the condition (CC), $\partial z-(\partial z)^{2} q \in C$ for some $0 \neq q \in C$. So $\partial w-(\partial w)^{2} \in C$, where $w=q z$, and $\partial w \notin C \partial y$. Again by the condition (CC), $\partial(y-w)-$ $(\partial(y-w))^{2} q_{1} \in C$ and $\partial(y+w)-(\partial(y+w))^{2} q_{2} \in C$ where $q_{1}, q_{2} \in C$. From these and the fact that $\partial y-(\partial y)^{2} \in C, \partial w-(\partial w)^{2} \in C$, one can easily see that $\left(q_{1}+q_{2}-2 q_{1} q_{2}\right) \partial y+\left(q_{1} q_{2}-2 q_{1} q_{2}\right) \partial w \in C$. Since $\partial w \notin C \partial y$, $q_{1}-q_{2}-2 q_{1} q_{2}=0$ and $q_{1}+q_{2}-2 q_{1} q_{2}=0$. Thus, $q_{1}=q_{2}=0$ and hence $\partial(y-w) \in C \cap \partial R$. Consequently $\partial(y-w)=0$, i.e., $\partial w=\partial y \in$ $C \partial y$, a contradiction. Therefore $\partial(R) \subseteq C$.

Case 2. $\partial R \cap C \neq\{0\}$. Suppose to the contrary that $\partial R \nsubseteq C$. Then as in Case 1 , there exists $y \in R$ such that $\partial y \notin C$ and $\partial y-$ $(\partial y)^{2} \in C$. So $(\partial y-1 / 2)^{2}=(\partial y)^{2}-\partial y+1 / 4 \in C$. Let $0 \neq \partial u \in \partial R \cap C$ and $t=1 / 2 u(\partial u)^{-1}$. Then $\partial t=1 / 2$ and $(\partial(y-t))^{2} \in C$. Hence by the condition (CC), $\partial(y-t) \in C$, i.e., $\partial y-1 / 2 \in C$. So $\partial y \in C$, again a contradiction. Hence $\partial R \cong C$. This completes the proof.

Using a similar proof of Theorem 4.5 we have

THeOREM 5.2. Let $R$ be a ring having a primary class $\mathscr{D}$ of derivations which satisfies the condition (CC). Then $\partial x \in C$ for all $\partial \in \mathscr{D}$ and $x \in R$.

6. Some remarks and open problems. In [1], we exhibited examples of primary class of derivations which are much smaller than the class $\mathscr{J}$ of all inner derivations. There we raised the problem on the existence of primary class of derivations $\mathscr{D}$ such that $\mathscr{D} \nsubseteq \mathscr{F}$. Now, we will present such an example.

EXAMPLE. Let $R$ be the subring of the ring of $2 \times 2$ matrices over $\approx$ which consists of all matrices of the form $\left(\begin{array}{cr}2 x & 2 y \\ 0 & 0\end{array}\right)$, where $x, y \in \mathscr{Z}$. The prime ideals of $R$ are of the form $\left(\begin{array}{lr}0 & 2 \mathscr{Z} \\ 0 & 0\end{array}\right)$ or $\left(\begin{array}{cc}2 p \not \mathscr{Z} & 2 \mathscr{Z} \\ 0 & 0\end{array}\right)$, where $p$ is an odd prime number in $\mathscr{r}$. The center of $R$ is $\left\{\left(\begin{array}{ll}0 & 0 \\ 0 & 0\end{array}\right)\right\}$. Let $\mathscr{D}$ be the set of all derivations $\partial$ on $R$ defined by

$$
\partial\left(\begin{array}{rr}
2 x & 2 y \\
0 & 0
\end{array}\right)=\left[\left(\begin{array}{rr}
0 & 2 a \\
0 & b
\end{array}\right),\left(\begin{array}{rr}
2 x & 2 y \\
0 & 0
\end{array}\right)\right]
$$

where $a, b \in \mathscr{K}$. It is easy to verify that $\mathscr{D}$ forms a primary class of derivations on $R$ and that, for $b \neq 0, \partial$ is not an inner derivation on $R$. 
There are still a lot of open problems concerning the commutativity of rings and primary class of derivations. We conclude with some of these problems.

Suppose $R$ is a ring having a primary class $\mathscr{D}$ of derivations.

(1) Suppose, for each $\partial \in \mathscr{D}$ and $x \in R$, there exist $m \in \mathscr{Z}^{+}$and $p \in R$ such that $(\partial x)^{m}-(\partial x)^{m+1} p \in C$, the center of $R$. What can we say about $R$ ? More precisely, do the nilpotent elements of $R$ form an ideal in $R$ ?

(2) Suppose $R$ is a division ring, and suppose, for each $\partial x \in$ $\mathscr{D}(R)$, there exists $n \in \mathscr{L}^{+}$such that $(\partial x)^{n} \in C$, the center of $R$. Is the dimension of $R$ considered as a vector space over $C$ atmost 4 ? The answer is affirmative if $\mathscr{D}$ is the primary class of all inner derivations on $R$ (see [5]).

(3) Can the results in the present paper also be extended to d.g. near rings? Some special cases have been done by Ligh [8].

(4) What kind of ring $R$ such that all its primary classes of derivations are contained in the class $\mathscr{F}$ of all inner derivations?

ACKNowledgment. The authors wish to express sincere thanks to the referee for his valuable suggestions.

Added in proof. In this paper "Nilpotent derivation" (Technion Preprint Series No. MT 453), Amos Kovacs has obtained independently an example of rings having a drimary class of derivations which is not contained in the class of all inner derivations.

\section{REFERENCES}

1. L. O. Chung, J. Luh and A. N. Richoux, Derivations and commutativity of rings, Pacific J. Math., 80 (1979), 77-89.

2. I. N. Herstein, The structure of a certain class of rings, Amer. J. Math., 75 (1953), $864-871$.

3. _ A condition for the commutativity of rings, Canad. J. Math., 9 (1957), $583-586$.

4. —— Noncommutative Rings, John Wiley, N. Y., 1968.

5. I. N. Herstein, C. Procesi and M. Schacher, Algebraic valued functions on noncommutative rings, J. of Algebra, 36 (1975), 128-150.

6. N. Jacobson, Structure theory for algebraic algebras of bounded degree, Ann. of Math., 46 (1945), 695-707.

7. — Structure of Rings, Amer. Math. Soc. colloquium Publ. 37, rev. ed., Providence, 1964.

8. S. Ligh, The structure of certain classes of rings and near rings, J. London Math. Soc., (2), 12 (1975), 27-31.

9. W. S. Martindale, III, The commutativity of a special class of rings, Canad. J. Math., 12 (1960), 263-268.

10. E. C. Posner, Derivations in prime rings, Proc. Amer. Math. Soc., 8 (1957), 1093-1100. 
11. M. S. Putcha, R. S. Wilson and A. Yaqub, Structure of rings satisfying certain identities on commutators, Proc. Amer. Math. Soc., 32 (1972), 57-62.

Received October 24, 1978 and in revised form April 25, 1979.

North Carolina State University

RALEIGH, NC 27650 


\section{PACIFIC JOURNAL OF MATHEMATICS}

\section{EDITORS}

DONALD BABBITT (Managing Editor)

University of California

Los Angeles, California 90024

HUgo RossI

University of Utah

Salt Lake City, UT 84112

C. C. MOORE and ANDREW OGG

University of California

Berkeley, CA 94720

\section{J. DUGUNDJI}

Department of Mathematics University of Southern California Los Angeles, California 90007

R. Finn and J. Milgram Stanford University Stanford, California 94305

\section{ASSOCIATE EDITORS}

E. F. BECKENBACH

B. H. NEUMANN

F. WOLF

K. YoshidA

\section{SUPPORTING INSTITUTIONS}

UNIVERSITY OF BRITISH COLUMBIA CALIFORNIA INSTITUTE OF TECHNOLOGY UNIVERSITY OF CALIFORNIA MONTANA STATE UNIVERSITY UNIVERSITY OF NEVADA, RENO NEW MEXICO STATE UNIVERSITY OREGON STATE UNIVERSITY UNIVERSITY OF OREGON
UNIVERSITY OF SOUTHERN CALIFORNIA STANFORD UNIVERSITY UNIVERSITY OF HAWAII UNIVERSITY OF TOKYO UNIVERSITY OF UTAH WASHINGTON STATE UNIVERSITY UNIVERSITY OF WASHINGTON 


\section{Pacific Journal of Mathematics}

Vol. 85, No. $1 \quad$ September, 1979

Ralph Alexander, Metric averaging in Euclidean and Hilbert spaces...... 1

B. Aupetit, Une généralisation du théorème de Gleason-Kahane-Żelazko

pour les algèbres de Banach..............................

Lung O. Chung, Jiang Luh and Anthony N. Richoux, Derivations and

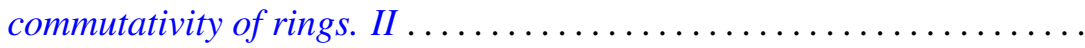

Lynn Harry Erbe, Integral comparison theorems for third order linear

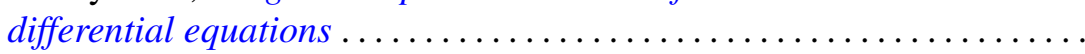

Robert William Gilmer, Jr. and Raymond Heitmann, The group of units of a

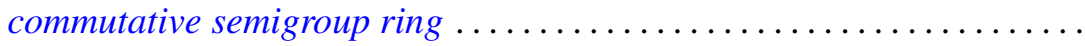

George Grätzer, Craig Robert Platt and George William Sands, Embedding lattices into lattices of ideals ...........................

Raymond D. Holmes and Anthony Charles Thompson, $n$-dimensional area and content in Minkowski spaces ....................... 77

Harvey Bayard Keynes and M. Sears, Modelling expansion in real flows....

Taw Pin Lim, Some classes of rings with involution satisfying the standard

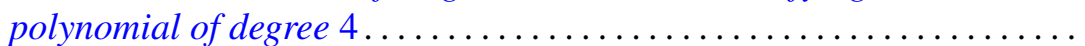

Garr S. Lystad and Albert Robert Stralka, Semilattices having bialgebraic congruence lattices ................................... 131

Theodore Mitchell, Invariant means and analytic actions . . . . . . . . . 145

Daniel M. Oberlin, Translation-invariant operators of weak type ........ 155

Raymond Moos Redheffer and Wolfgang V. Walter, Inequalities involving

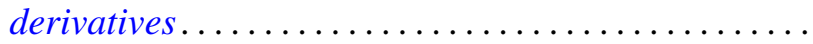

Eric Schechter, Stability conditions for nonlinear products and semigroups ................................

Jan Søreng, Symmetric shift registers ........................ 201

Toshiji Terada, On spaces whose Stone-Čech compactification is $\mathrm{Oz} \ldots \ldots .231$

Richard Vrem, Harmonic analysis on compact hypergroups ... 